05,12

\title{
Влияние магнитомягкой фазы на процессы перемагничивания магнитотвердого/магнитомягкого бислоя
}

\author{
(C) Т.А. Тааев, К.Ш. Хизриев, А.К. Муртазаев
}

Институт фозики им. Х.И. Амирханова ДФИЦ РАН, Махачкала, Россия

E-mail: taaev89@mail.ru

Поступила в Редакцию 30 декабря 2019 г.

В окончательной редакции 30 декабря 2019 г.

Принята к публикации 10 января 2020 г.

\begin{abstract}
Проведено исследование процессов перемагничивания магнитотвердого/магнитомягкого бислоя во внешнем магнитном поле с помощью метода Монте-Карло. Построены кривые перемагничивания магнитного бислоя при различных значениях толщины магнитомягкого слоя. Также изучено влияние величины внутрислойного обменного взаимодействия и константы анизотропии магнитомягкого слоя на процессы перемагничивания. Приведены фазовые диаграммы магнитного бислоя.
\end{abstract}

Ключевые слова: магнетизм, спиновая пружина, магнитотвердая/магнитомягкая гетероструктура, метод Монте-Карло.

DOI: $10.21883 /$ FTT.2020.06.49335.29M

\section{1. Введение}

С точки зрения современной реальности развития электронных нанотехнологий чрезвычайно интересными объектами являются обменно-связанные магнитные гетероструктуры, состоящие из чередующихся магнитотвердых и магнитомягких атомных слоев [1-22]. Стремительный рост интереса к этим искусственным магнитным материалам связан не только с широкими перспективами их практического применения, но и с тем, что они обладают рядом уникальных особенностей. Например, они открывают возможности для увеличения максимального энергетического произведения $(B H)_{\max }$, а также в них возможно формирование во внешнем магнитном поле гетерофазной одномерной спиновой пружины. Совсем недавно были получены магнитные гетероструктуры, в которых образуются устойчивые спиновые пружины [23]. Поэтому технологическая значимость этих материалов приобретает новый уровень: использование в качестве быстродействующих аттенюаторов, магнитных датчиков, продвинутых носителей информации с большой плотностью записи и т.д.

Основной характеристикой магнитотвердых материалов является максимальное значение энергетического произведения $(B H)_{\max }$. Показатель $(B H)_{\max }$ определяется высокими значениями намагниченности насыщения материала, магнитной анизотропии и температуры Кюри. Большими значениями намагниченности насыщения обладают магнитомягкие материалы, такие как железо, кобальт. А редкоземельные материалы (Re) обладают сильной анизотропией и имеют большую магнитную жесткость. Магнитный бислой, состоящий из магнитомягкого (Fe, Со и др.) и магнитотвердого слоя $(\mathrm{Re})$, будет иметь большие значения энергетического произведения $(B H)_{\max }$. В настоящей работе мы исследуем влия- ние толщины, значений обменного внутрислойного взаимодействия и константы анизотропии магнитомягкого слоя на процессы перемагничивания модели магнитного бислоя методом Монте-Карло.

\section{2. Модель и методика исследования}

Исследование $\mathrm{SmCo} / \mathrm{Fe}$ бислоя методом мёссбауэровской спектроскопии, характеризующихся большим значением анизотропии типа „легкая ось“, показало упорядочение магнитных моментов в плоскости системы [13]. В связи с этим, для изучения системы магнитотвердого/магнитомягкого бислоя мы используем стандартную ХҮ-модель [24-27].

Гамильтониан системы имеет вид:

$$
\begin{aligned}
H= & -\frac{1}{2} \sum_{i, j} J\left(S_{i}^{x} S_{j}^{x}+S_{i}^{y} S_{j}^{y}\right)-\sum_{i} K\left(S_{i}^{x}\right)^{2} \\
& -g \mu \sum_{i}\left(H_{0}^{x} S_{i}^{x}+H_{0}^{y} S_{i}^{y}\right),
\end{aligned}
$$

где первая сумма учитывает обменное взаимодействие каждого магнитного атома с ближайшими соседями внутри слоев с обменами $J=J_{\text {hard }}$ и $J=J_{\text {soft }}$ в магнитотвердом и магнитомягком слоях соответственно, и межслойное взаимодействие с параметром $J=J_{\text {int }}$; вторая сумма учитывает вклад анизотропии в энергию системы, $K=K_{\text {hard }}$ и $K=K_{\text {soft }}-$ соответственно константы анизотропии магнитотвердого и магнитомягкого слоев; третья сумма - вклад внешнего магнитного поля в энергию системы, $g \approx 2-$ фактор Ланде, $\mu-$ магнетон Бора, $H_{0}^{x, y}$ - проекции вектора внешнего магнитного поля $\mathbf{H}_{0}, S_{i}^{x, y}-$ проекции спина $\mathbf{S}_{i}$, локализованного на узле $i,\left|\mathbf{S}_{i}\right|=1$. 
Расчеты проводились стандартным алгоритмом Метрополиса метода Монте-Карло [28] для систем с линейными размерами $L x \times L y \times L z$, при температуре $T$ намного ниже $T_{c}$ для модели магнитного бислоя [25]. По осям $x$ и $y$ линейные размеры системы были фиксированы $(L x=L y=50)$, а по оси $z$ принимали различные значения, в зависимости от толщины магнитомягкого слоя. Толщина магнитотвердого слоя была фиксирована $\left(t_{\text {hard }}=50\right)$, а магнитомягкого слоя варьировала от 10 до 50 атомных слоев.

В ходе численного эксперимента нами были использованы следующие значения параметров: $J_{\text {soft }}=2.8 \cdot 10^{-6} \mathrm{ergs} / \mathrm{cm}, J_{\text {hard }}=1.2 \cdot 10^{-6} \mathrm{ergs} / \mathrm{cm}, J_{\text {int }}=$ $=1.8 \cdot 10^{-6} \mathrm{ergs} / \mathrm{cm}, \quad K_{\text {hard }} \times d^{2} / J_{\text {soft }}=7.14 \cdot 10^{-3}$, $K_{\text {soft }}=0, d=2 \AA-$ расстояние между атомными слоями, $\left|\mathbf{S}_{i}\right|=1[2,25]$. В гамильтониане (1) значения обменных констант и констант анизотропии были нормированы $\kappa J_{\text {soft }}$ Угол между направлениями векторов внешнего магнитного поля и анизотропии магнитотвердого слоя равен $177^{\circ}$.

В процессе численного эксперимента наблюдали за следующими параметрами: намагниченностью всей системы $M$, ее продольными $M_{\text {long }}$ и поперечными $M_{\text {tran }}$ компонентами, а также за намагниченностями каждого магнитного монослоя $M_{j}$ :

$$
\begin{gathered}
M=\left\langle\frac{1}{N} \sum_{i=1}^{N} \mathbf{S}_{i}\right\rangle, \\
M_{\text {long }}=M \cos \alpha, \\
M_{\text {tran }}=M \sin \alpha, \\
M_{j}=\left\langle\frac{1}{L x \times L y} \sum_{i=1}^{L x \times L y}\right\rangle_{j},
\end{gathered}
$$

где $N$ - число атомов, $\alpha$ - угол между направлениями векторов намагниченности $M$ и анизотропии магнитотвердого слоя типа „легкая ось“. Угловые скобки означают усреднение по ансамблю.

\section{3. Результаты исследования}

На рис. 1 показаны полевые зависимости общей намагниченности $M$ при различной толщине $t_{\text {soft }}$ магнитомягкого слоя. Как видно на рис. 1 с увеличением толщины магнитомягкого слоя, характеристические значения $H_{\mathrm{ex}}$ (поле обменного смещения, при котором магнитные моменты атомов магнитомягкого слоя начинают вращаться, как в стенке Блоха) и $H_{\text {irr }}$ (критическое поле) внешнего магнитного поля уменьшаются. Это связано с тем, что в модели магнитного бислоя анизотропия магнитомягкого слоя равна нулю и магнитные моменты в этом слое свободно вращаются под действием внешнего магнитного поля. Магнитомягкий слой ухудшает магнитоупругие свойства магнитного бислоя.

На рис. 2 приведена фазовая диаграмма зависимости значений $H_{\mathrm{ex}}$ и $H_{\mathrm{irr}}$ от толщины магнитомягкого слоя.

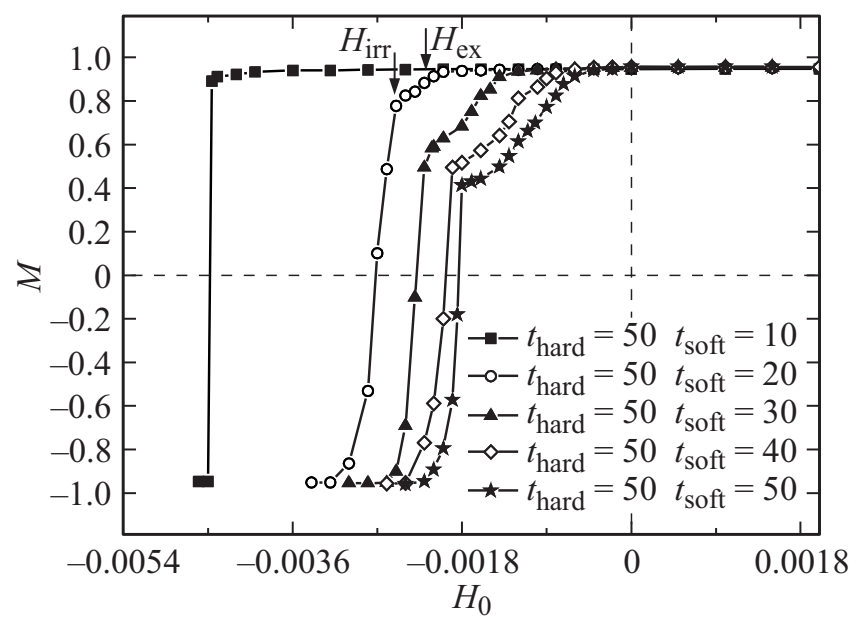

Рис. 1. Кривые перемагничивания общей намагниченности $M$ при различной толщине магнитомягкого слоя $t_{\text {soft. }}$.

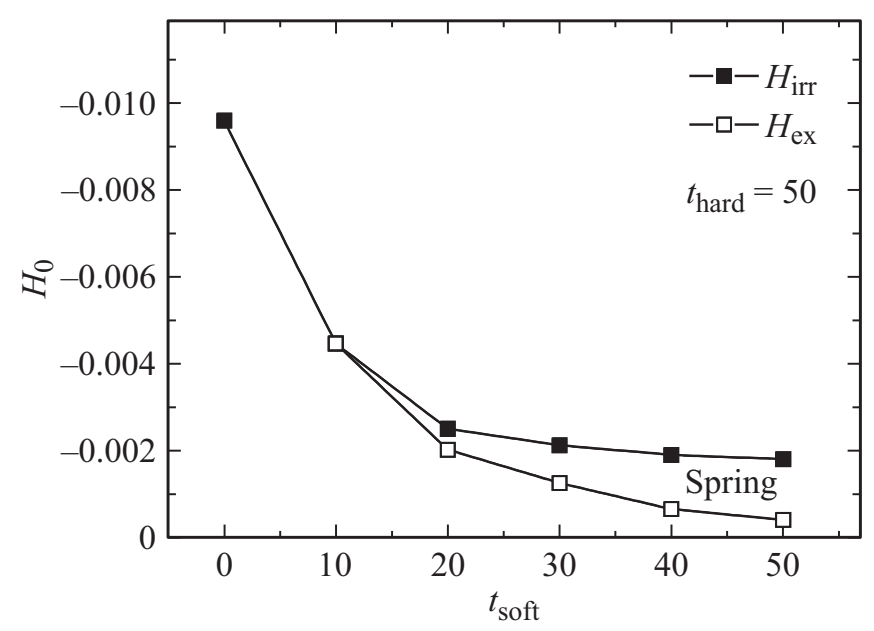

Рис. 2. Фазовая диаграмма зависимости значений $H_{\text {ex }}$ и $H_{\text {irr }}$ внешнего магнитного поля от толщины магнитомягкого слоя $t_{\text {soft }}$.

„Spring“ - область между кривыми $H_{\mathrm{ex}}$ и $H_{\text {irr }}$, где в магнитном бислое образуется спиновая пружина при включении внешнего магнитного поля. С уменьшением толщины магнитомягкого слоя $t_{\text {soft }}$ величины $H_{\mathrm{ex}}$ и $H_{\text {irr }}$ стремятся к одному значению, область „spring“ уменьшается, а при толщине магнитомягкого слоя в 10 атомных слоев и вовсе исчезает. Причиной этому является то, что при толщине $t_{\text {soft }} \leq 10$ магнитные моменты атомов магнитомягкого слоя жестко связаны с магнитными моментами атомов магнитотвердого слоя и практически не вращаются. В связи с чем, спиновая пружина в модели магнитного бислоя не образуется.

Также на рис. 2 приведено значение критического магнитного поля $H_{\text {irr }}$ для магнитотвердого слоя. Как видно из рисунка при добавлении магнитомягкого слоя толщиной всего лишь 10 атомных монослоев, значение $H_{\text {irr }}$ уменьшается практически вдвое. Зависимость критического значения внешнего магнитного поля $H_{\text {irr }}$ 


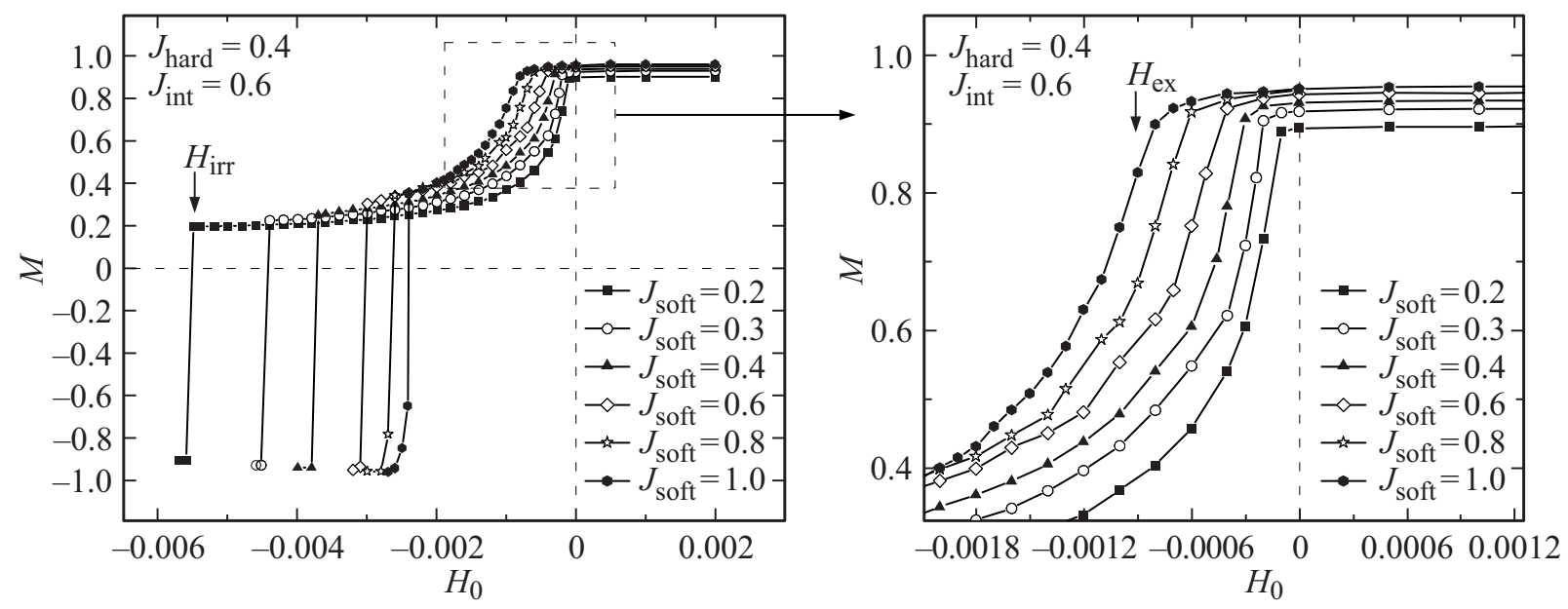

Рис. 3. Кривые перемагничивания общей намагниченности $M$ при различных значениях константы обменного параметра $J_{\text {soft. }}$

от толщины магнитомягкого слоя хорошо согласуется с результатами работ Фуллертона и его коллег [2].

Из работы [4] известно, что во внешнем магнитном поле намагниченность магнитомягкого слоя остается сонаправленной и параллельной намагниченности магнитотвердого слоя вплоть до значений внешнего поля

$$
H_{\mathrm{ex}}=\pi^{2} J_{\mathrm{soft}} / 2 M_{s} t_{\mathrm{soft}}^{2},
$$

где $J_{\text {soft }}$ - обменная константа взаимодействия между спинами внутри магнитомягкого слоя, $t_{\mathrm{soft}}$ - толщина магнитомягкого слоя, $M_{s}$ - намагниченность насыщения магнитомягкого слоя.

Как следует из формулы (6) значение поля обменного смещения $H_{\mathrm{ex}}$ имеет обратную квадратичную зависимость от толщины магнитомягкого слоя $\left(H_{\mathrm{ex}} \propto 1 / t_{\mathrm{soft}}^{2}\right)$. На рис. 2 видно, что наши результаты качественно хорошо согласуется с теоретическими предсказаниями при толщине магнитомягкого слоя $t_{\text {soft }}>20$ атомных слоев.

На рис. 3 показаны кривые перемагничивания магнитной модели при различных значениях константы обменного взаимодействия магнитомягкого слоя $J_{\text {soft }}$. Значения обменных констант магнитотвердого слоя и межслойного взаимодействия принимали значения $J_{\text {hard }}=0.4$ и $J_{\text {int }}=0.6$. Толщина магнитотвердого и магнитомягкого слоев были фиксированы $\left(t_{\mathrm{soft}}=t_{\text {hard }}=50\right.$ атомных монослоев). Как видно на рис. 3 , при увеличении константы обменного взаимодействия магнитомягкого слоя $J_{\text {soft }}$, критическое значение $H_{\text {irr }}$ внешнего магнитного поля уменьшается, а значение $H_{\mathrm{ex}}-$ растет. Увеличение константы $J_{\text {soft }}$ приводит к тому, что магнитные моменты атомов более жестко связаны между собой и, соответственно, требуется приложить большую величину магнитного поля, чтобы заставить их вращаться. Поле обменного смещения $H_{\mathrm{ex}}$ увеличивается, и петля становится более выпуклой. Увеличение внутрислойного обмена приводит к тому, что в результате поворота магнитные моменты магнитомягкого слоя увлекают за

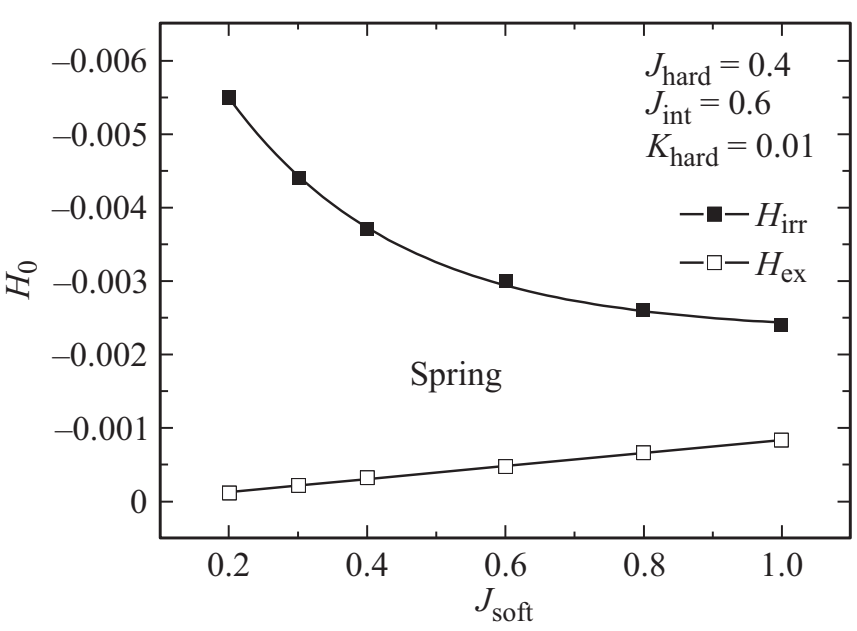

Рис. 4. Фазовая диаграмма зависимости характеристических значений магнитных полей $H_{\text {irr }}$ и $H_{\text {ex }}$ от константы обменного взаимодействия магнитомягкого слоя $J_{\text {soft }}$.

собой спины всё большего количества соседних монослоев и намагниченность магнитомягкого слоя быстро достигает насыщения.

Зависимость величин $H_{\text {irr }}$ и $H_{\text {ex }}$ от значения обменного параметра магнитомягкого слоя приведена на рис. 4. Из формулы (6) также видно, что характеристическое значение поля $H_{\text {ex }}$ зависит линейно от обменного взаимодействия магнитомягкого слоя $J_{\text {soft }}\left(H_{\text {ex }} \propto J_{\text {soft }}\right)$. В рамках погрешности, значения $H_{\mathrm{ex}}$ практически ложатся на прямую, что свидетельствует о хорошем согласии наших данных с формулой (6). Более детальное исследование влияния обменных параметров внутрислойного и межслойного взаимодействия модели магнитотвердого/магнитомягкого бислоя было выполнено в работе [24].

Полевые зависимости общей намагниченности $M$ при различных значениях константы анизотропии $K_{\text {soft }}$ магнитомягкого слоя показаны на рис. 5. Значения остальных параметров обменного взаимодействия дан- 


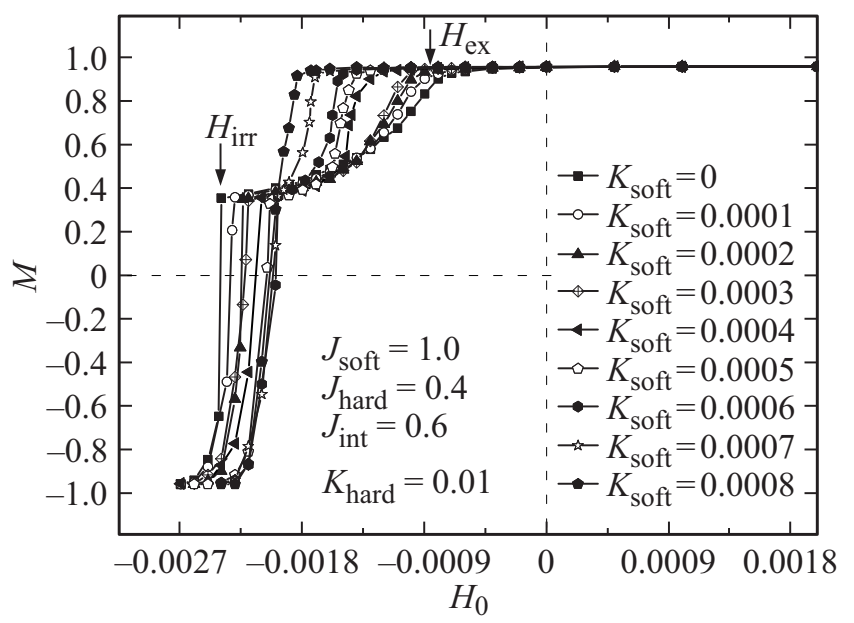

Рис. 5. Кривые перемагничивания общей намагниченности $M$ при различных значениях константы анизотропии магнитомягкого слоя $K_{\text {soft }}$.

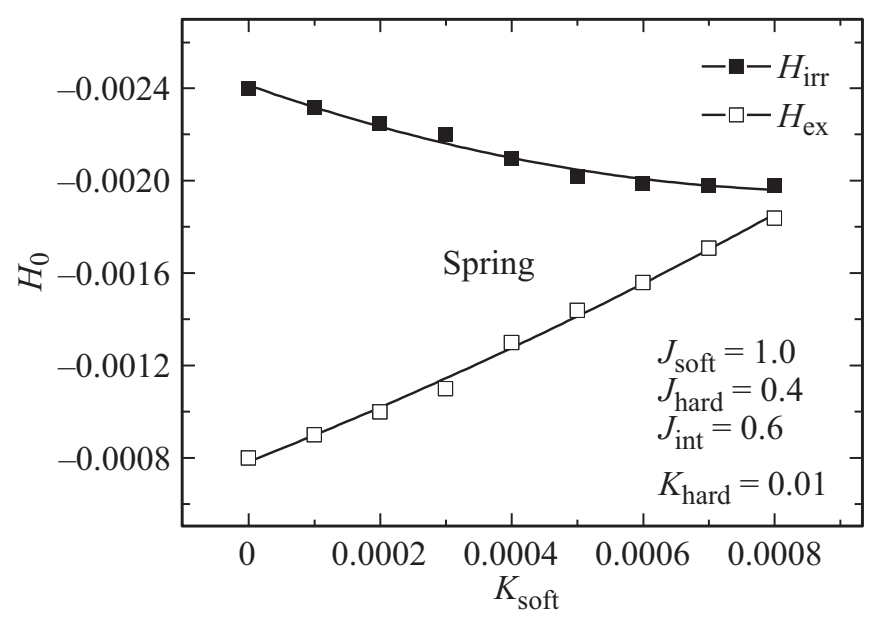

Рис. 6. Фазовая диаграмма зависимости характеристических значений магнитных полей $H_{\text {irr }}$ и $H_{\text {ex }}$ от константы магнитомягкого слоя $K_{\text {soft }}$.

ной модели магнитного бислоя были фиксированы. Как видно из рисунка, увеличение константы анизотропии магнитомягкого слоя влечет за собой увеличение поля обменного смещения $H_{\mathrm{ex}}$ и уменьшение критического поля $H_{\text {irr. }}$ Появление анизотропии типа „легкая ось“ в магнитомягком слое приводит к тому, что магнитные моменты атомов принимают только два положения: против и вдоль приложенного внешнего магнитного поля. В связи с чем, магнитные моменты атомов не будут свободно вращаться под действием внешнего магнитного поля. Это вызывает увеличение поля обменного смещения $H_{\text {ex }}$ и уменьшение критического поля $H_{\text {irr. }}$.

На рис. 6. приведена зависимость характеристических значений $H_{\text {ex }}$ и $H_{\text {irr }}$ от константы анизотропии $K_{\text {soft }}$ магнитомягкого слоя. Область „spring“ на фазовой диаграмме (рис. 6) с ростом $K_{\text {soft }}$ уменьшается, а при значении $K_{\text {soft }}=0.0008$ практически исчезает. Поведение магнитомягкого слоя во внешнем магнитном поле становится похожим на поведение магнитотвердого слоя. Критическое значение константы анизотропии магнитомягкого слоя $\left(K_{\text {soft }}=0.0008\right)$, при котором в магнитном бислое невозможно образование спиновой пружины, на порядок меньше константы анизотропии $K_{\text {hard }}$ магнитотвердого слоя.

\section{4. Заключение}

В работе показано, что изменением некоторых параметров магнитомягкого слоя можно добиться получения магнитотвердого/магнитомягкого бислоя с необходимыми, наперед заданными и уникальными магнитными характеристиками. В частности, было изучено влияние трех параметров магнитомягкого слоя на магнитные свойства бислоя: значения толщины магнитомягкого слоя, величины константы обменного взаимодействия и константы анизотропии магнитомягкого слоя. Увеличение толщины магнитомягкого слоя при фиксированной толщине магнитотвердого слоя приводит к уменьшению характеристических значений $H_{\mathrm{ex}}$ и $H_{\text {irr }}$ внешнего магнитного поля и магнитоупругие свойства ухудшаются. Зависимость значения $H_{\mathrm{ex}}$ от толщины магнитомягкого слоя хорошо согласуется с теоретическими выкладками при толщине магнитомягкого слоя больше 20 атомных слоев. Показано, что поле обменного смещения $H_{\text {ex }}$ зависит линейно от $J_{\text {soft }}$, что хорошо согласуется с теоретическими данными. Появление анизотропии в магнитомягком слое улучшает магнитоупругие свойства магнитного бислоя. Но при значении $K_{\text {soft }} \geq 0.0008$, магнитомягкий слой становится по своим магнитоупругим свойствам похожим на магнитотвердый и образование спиновой пружины в модели магнитотвердого/магнитомягкого бислоя невозможно. Опираясь на результаты исследования, можно будет определить оптимальный состав для производства подходящих магнитотвердых/магнитомягких гетероструктур.

\section{Финансирование работы}

Работа выполнена при финансовой поддержке Российского фонда фундаментальных исследований (грант № 18-32-00526, ,мол_а“ ${ }^{\text {“6). }}$

\section{Конфликт интересов}

Авторы заявляют, что у них нет конфликта интересов.

\section{Список литературы}

[1] E.F. Kneller, R. Hawig. IEEE Trans. Magn. 27, 3588 (1991).

[2] E.E. Fullerton, J.S. Jiang, M. Grimsditch, C.H. Sowers, S.D. Bader. Phys. Rev. B 58, 12193 (1998).

[3] R. Skomski, J.M.D. Coey. Phys. Rev. B 48, 15812 (1993).

[4] E. Goto, N. Hayashi, T. Miyashita, K. Nakagawa. J. Appl. Phys. 36, 2951 (1965).

[5] K. Mibu, T. Nagahama, T. Shinjo. JMMM 163, 75 (1996). 
[6] R. Coehoorn, D.B. de Mooij, C. De Waard. JMMM 80, 101 (1989).

[7] M. Amato, M.G. Pini, A. Rettori. Phys. Rev. B 60, 3414 (1999).

[8] R. Andreescu, M.J. O’Shea. J. Appl. Phys. 91, 8183 (2002).

[9] Shi-Shen Yan, M. Elkawni, D.S. Li, H. Garmestani, J.P. Liu, J.L. Weston, G. Zangari. J. Appl. Phys. 94, 4535 (2003).

[10] Guang-hua Guo, Guang-fu Zhang, San-yuan Song, D.W. Wang, G.J. Bowden, P.A.J. de Groot. Appl. Phys. Lett. 93, 102505 (2008)

[11] F. Montaigne, S. Mangin, Y. Henry. Phys. Rev. B 67, 144412 (2003).

[12] J. McCord, Y. Henry, T. Hauet, F. Montaigne, E.E. Fullerton, S. Mangin. Phys. Rev. B 78, 094417 (2008).

[13] V.M. Uzdin, A. Vega, A. Khrenov, W. Keune, V.E. Kuncser, J.S. Jiang, S.D. Bader. Phys. Rev. B 85, 024409 (2012).

[14] Cai-yin You, ChoongJin Yang, Z.D. Zhang, JongSoo Han, X.K. Sun. J. Mater. Res. 19, 786 (2004).

[15] V.M. Uzdin, A. Vega. Nanotechnology 19, 315401 (2008).

[16] A.V. Khvalkovskii, K.A. Zvezdin, A.A. Zvezdin, V.S. Gornakov, D.G. Skachkov, P. Perlo. Physica B 372, 358 (2006).

[17] N. de Sousa, A. Apolinario, F. Vernay, P.M.S. Monteiro, F. Albertini, F. Casoli, H. Kachkachi, D.S. Schmool. Phys. Rev. B 82, 104433 (2010).

[18] P. Chowdhury, M. Krishnan, Harish C. Barshilia, D.V. SridharaRao, Deepak Kumar, C. Shivakumara. JMMM 342, 74 (2013).

[19] S. Djedai, E. Talbot, P.E. Berche. JMMM 368, 29 (2014).

[20] Rajan Goyal, Nishta Arora, Akanksha Kapoor, S. Lamba, S. Annapoorni. J. Alloys Comp. 695, 1014 (2017).

[21] Г.С. Патрин, И.А. Турпанов, В.И. Юшков, А.В. Кобяков, К.Г. Патрин, Г.Ю. Юркин, Я.А. Живая. Письма в ЖЭТФ 109, 324 (2019).

[22] A.V. Kobyakov, I.A. Turpanov, G.S. Patrin, V.I. Yushkov, S.A. Yarikov, M.N. Volochaev, Ya.A. Zhivaya. J. Phys. Conf. Ser. 1389, 012028 (2019).

[23] L.V. Dzemiantsova, G. Meier, R. Röhlsberger. Sci. Rep. 5, 16153 (2015).

[24] Т.А. Тааев, К.Ш. Хизриев, А.К. Муртазаев. ЖЭТФ 156, 331 (2019).

[25] T.A. Taaev, K.Sh. Khizriev, A.K. Murtazaev, V.M. Uzdin. J. Alloys Comp. 678, 167 (2016).

[26] Т.А. Тааев, К.Ш. Хизриев, А.К. Муртазаев. ЖЭТФ 151, 1084 (2017).

[27] T.A. Taaev, K.Sh. Khizriev, A.K. Murtazaev. J. Alloys Comp. 785, 1253 (2019).

[28] N. Metropolis, A.W. Rosenbluth, M.N. Rosenbluth, A.H. Teller, E. Teller. J. Chem. Phys. 21, 1087 (1953).

Редактор Т.Н. Василевскал 